\title{
THE LATTICE AND SIMPLEX STRUCTURE OF STATES ON PSEUDO EFFECT ALGEBRAS
}

\author{
ANATOLIJ DVUREČENSKIJ \\ 1 Mathematical Institute, Slovak Academy of Sciences \\ Štefánikova 49, SK-814 73 Bratislava, Slovakia \\ E-mail: dvurecen@mat.savba.sk,
}

\begin{abstract}
We study states, measures, and signed measures on pseudo effect algebras with some kind of the Riesz Decomposition Property, (RDP). We show that the set of all Jordan signed measures is always an Abelian Dedekind complete $\ell$-group. Therefore, the state space of the pseudo effect algebra with (RDP) is either empty or a nonempty Choquet simplex or even a Bauer simplex. This will allow represent states on pseudo effect algebras by standard integrals.
\end{abstract}

\section{INTRODUCTION}

The seminal paper by Birkhoff and von Neumann BiNe showed that the events of quantum mechanical measurements do not fulfill the axioms of Boolean algebras and therefore also do not axioms of the classical probability theory presented by Kolmogorov [Kol]. It initiated the research of the mathematical foundations of quantum physics. Nowadays, there appeared a whole hierarchy of so-called quantum structures, like orthomodular lattices and posets, orthoalgebras, etc. Since the Nineties, we are intensively studying effect algebras that were introduced by Foulis and Bennett $\mathrm{FoBe}$. An extensive source of information about effect algebras can be found in $\mathrm{DvPu}$. Orthodox examples of the Hilbert space quantum mechanics are the system of closed subspaces, $\mathcal{L}(H)$, of a Hilbert space $H$ (real, complex or quaternionic) and the system of all Hermitian operators, $\mathcal{E}(H)$, that are between the zero operator and the identity operator. An effect algebra $E$ is a partial algebraic structure with a partially defined binary operation, + , that is commutative and it models join of "mutually exclusive" events. In many cases, it is an interval in a po-group (= partially ordered group), like $\mathcal{E}(H)$ is an interval in the po-group $\mathcal{B}(H)$ of all Hermitian operators on a Hilbert space $H$. A sufficient condition for an effect algebra to be an interval is e.g. the Riesz Decomposition Property (RDP, for short); and in such a case, $E$ is an interval in a unique unital Abelian po-group $(G, u)$ with interpolation, or equivalently, with (RDP), see $\mathrm{Rav}$ or $\mathrm{DvPu}$, Thm 1.7.17].

\footnotetext{
${ }^{1}$ Keywords: Pseudo effect algebra; effect algebra; Riesz Decomposition Properties; signed measure, state; Jordan signed measure, unital po-group; simplex; Choquet simplex; Bauer simplex AMS classification: 81P15, 03G12, 03B50

The author thanks for the support by Center of Excellence SAS - Quantum Technologies -, ERDF OP R\&D Projects CE QUTE ITMS 26240120009 and meta-QUTE ITMS 26240120022, the grant VEGA No. 2/0032/09 SAV.
} 
In the last decade, there appeared many structures where the basic operation, + , is not necessarily commutative. The papers DvVe1, DvVe2 present a noncommutative generalization of effect algebras, called pseudo effect algebras. In some important examples, they are also an interval in a unital po-group but not necessarily Abelian. Sufficient conditions for a pseudo effect algebra to be an interval in a unital po-group are stronger versions of (RDP), see DvVe2 for more details.

Any measurement is accomplished by probabilistic reasoning. The quantum mechanical one is described by a state, an analogue of a probability measure. The state space of any pseudo effect algebra is an interesting structure that can be also void, see e.g. Dvu1, but in general it is a convex compact Hausdorff topological space. In very important cases, it is a simplex and this allows then characterize states via an integral through a regular Borel probability measure, in some cases even in a unique way, see Dvu3].

If an effect algebra satisfies (RDP), then it is an interval in an Abelian unital po-group with interpolation (RIP), so that it is a non-void simplex, Dvu2, Thm 5.1]. If $E$ is a pseudo effect with (RDP) that is an interval in a unital po-group, then it can happen that the state space is empty, [Dvu1]. In [Dvu3, Thm 4.2], we have showed that every interval pseudo effect algebra with (RDP) or an effect algebra with $(\mathrm{RDP})_{1}$ is a simplex.

The Riesz Decomposition Property is a weaker form of distributivity - it allows to make a joint refinement of two decompositions of the unit element. This is a reason why $(\mathrm{RDP})$ fails to hold for $\mathcal{L}(H)$ and $\mathcal{E}(H)$.

We do not know whether every pseudo effect algebra with (RDP) is an interval in a unital po-group, this is known only for a stronger version $(\mathrm{RDP})_{1}$, [DvVe2, Thm 5.7]. Hence, we cannot directly apply the result from [Dvu3, Thm 4.2]. Therefore, we prove in the paper that the state space of a pseudo effect algebra with (RDP) is empty or a non-void Choquet simplex, Theorem 5.1. To prove that, we are studying the set of Jordan signed measures on a pseudo effect algebra with (RDP). We show that such a set is either a singleton containing only the zero measure or it is a non-trivial Abelian Dedekind complete $\ell$-group (= lattice ordered). The simplex structure will be finally applied to represent a state as an integral through a unique regular Borel probability measure. We note that such a representation of states for $\mathrm{MV}$-algebras (= effect algebras with $(\mathrm{RDP})_{2}=$ Phi-symmetric effect algebras, see BeFo] ) was proved in [Kro, Pan] and for effect algebras in Dvu3].

The paper is organized as follows.

The elements of pseudo effect algebras are presented in Section 2. Section 3 describes the lattice structure of the group of all Jordan signed measures on a pseudo effect algebra with (RDP). Section 4 will describe some basic properties of Jordan signed measures that were known only for classical measures. Applications of the simplex structures of the state space, Choquet or Bauer simplices, for representation of states by integral are given in Section 5. The final concluding remarks are presented in Section 6 .

\section{Pseudo Effect Algebras}

Following DvVe1, Dvu2, we say that a pseudo effect algebra is a partial algebra $(E ;+, 0,1)$, where + is a partial binary operation and 0 and 1 are constants, such that for all $a, b, c \in E$, the following holds 
(i) $a+b$ and $(a+b)+c$ exist if and only if $b+c$ and $a+(b+c)$ exist, and in this case $(a+b)+c=a+(b+c)$;

(ii) there is exactly one $d \in E$ and exactly one $e \in E$ such that $a+d=e+a=1$;

(iii) if $a+b$ exists, there are elements $d, e \in E$ such that $a+b=d+a=b+e$;

(iv) if $1+a$ or $a+1$ exists, then $a=0$.

If we define $a \leq b$ if and only if there exists an element $c \in E$ such that $a+c=b$, then $\leq$ is a partial ordering on $E$ such that $0 \leq a \leq 1$ for any $a \in E$. It is possible to show that $a \leq b$ if and only if $b=a+c=d+a$ for some $c, d \in E$. We write $c=a / b$ and $d=b \backslash a$. Then

$$
(b \backslash a)+a=a+(a / b)=b,
$$

and we write $a^{-}=1 \backslash a$ and $a^{\sim}=a / 1$ for any $a \in E$.

For basic properties of pseudo effect algebras see [DvVe1, DvVe2]. We recall that if + is commutative, $E$ is said to be an effect algebra; for a comprehensive overview on effect algebras see e.g. $\mathrm{DvPu}$. It is worthy to remark that effect algebras are equivalent to D-posets, where the basic operation is a difference of two comparable events, $\mathrm{KoCh}$.

We recall that a po-group (= partially ordered group) is a group $G$ with a partial order, $\leq$, such that if $a \leq b, a, b \in G$, then $x+a+y \leq x+b+y$ for all $x, y \in G$. We denote by $G^{+}$the set of all positive elements of $G$. If, in addition, $\leq$ implies that $G$ is a lattice, we call it an $\ell$-group (= lattice ordered group). An element $u \in G^{+}$is said to a strong unit if given $g \in G$, there is an integer $n \geq 1$ such that $g \leq n u$, and the couple $(G, u)$ with a fixed strong unit is said to a unital po-group. The monographs like [Fuc, Gla can serve as a basic source of information about partially ordered groups.

If $(G, u)$ is a unital (not necessary Abelian) po-group with strong unit $u$, and

$$
\Gamma(G, u):=\{g \in G: 0 \leq g \leq u\},
$$

then $(\Gamma(G, u) ;+, 0, u)$ is a pseudo effect algebra if we restrict the group addition + to the set of all those $(x, y) \in \Gamma(G, u) \times \Gamma(G, u)$ that $x \leq u-y$.

Every pseudo effect algebra $E$ that is isomorphic to some $\Gamma(G, u)$ is said to be an interval pseudo effect algebra.

According to DvVe1, we introduce for pseudo effect algebras the following forms of the Riesz Decomposition Properties which in the case of commutative effect algebras can coincide:

(a) For $a, b \in E$, we write $a \operatorname{com} b$ to mean that for all $a_{1} \leq a$ and $b_{1} \leq b, a_{1}$ and $b_{1}$ commute.

(b) We say that $E$ fulfils the Riesz Interpolation Property, (RIP) for short, if for any $a_{1}, a_{2}, b_{1}, b_{2} \in E$ such that $a_{1}, a_{2} \leq b_{1}, b_{2}$ there is a $c \in E$ such that $a_{1}, a_{2} \leq c \leq b_{1}, b_{2}$.

(c) We say that $E$ fulfils the weak Riesz Decomposition Property, $\left(\mathrm{RDP}_{0}\right)$ for short, if for any $a, b_{1}, b_{2} \in E$ such that $a \leq b_{1}+b_{2}$ there are $d_{1}, d_{2} \in E$ such that $d_{1} \leq b_{1}, d_{2} \leq b_{2}$ and $a=d_{1}+d_{2}$.

(d) We say that $E$ fulfils the Riesz Decomposition Property, (RDP) for short, if for any $a_{1}, a_{2}, b_{1}, b_{2} \in E$ such that $a_{1}+a_{2}=b_{1}+b_{2}$ there are $d_{1}, d_{2}, d_{3}, d_{4} \in$ $E$ such that $d_{1}+d_{2}=a_{1}, d_{3}+d_{4}=a_{2}, d_{1}+d_{3}=b_{1}, d_{2}+d_{4}=b_{2}$.

(e) We say that $E$ fulfils the commutational Riesz Decomposition Property, $\left(\mathrm{RDP}_{1}\right)$ for short, if for any $a_{1}, a_{2}, b_{1}, b_{2} \in E$ such that $a_{1}+a_{2}=b_{1}+b_{2}$ 
there are $d_{1}, d_{2}, d_{3}, d_{4} \in E$ such that (i) $d_{1}+d_{2}=a_{1}, d_{3}+d_{4}=a_{2}$, $d_{1}+d_{3}=b_{1}, d_{2}+d_{4}=b_{2}$, and (ii) $d_{2}$ com $d_{3}$.

(f) We say that $E$ fulfils the strong Riesz Decomposition Property, $\left(\mathrm{RDP}_{2}\right)$ for short, if for any $a_{1}, a_{2}, b_{1}, b_{2} \in E$ such that $a_{1}+a_{2}=b_{1}+b_{2}$ there are $d_{1}, d_{2}, d_{3}, d_{4} \in E$ such that (i) $d_{1}+d_{2}=a_{1}, d_{3}+d_{4}=a_{2}, d_{1}+d_{3}=b_{1}$, $d_{2}+d_{4}=b_{2}$, and (ii) $d_{2} \wedge d_{3}=0$.

We have the implications

$$
\left(\mathrm{RDP}_{2}\right) \Rightarrow\left(\mathrm{RDP}_{1}\right) \Rightarrow(\mathrm{RDP}) \Rightarrow\left(\mathrm{RDP}_{0}\right) \Rightarrow(\mathrm{RIP})
$$

The converse of any of these implications does not hold. For commutative effect algebras we have

$$
\left(\mathrm{RDP}_{2}\right) \Rightarrow\left(\mathrm{RDP}_{1}\right) \Leftrightarrow(\mathrm{RDP}) \Leftrightarrow\left(\mathrm{RDP}_{0}\right) \Rightarrow(\mathrm{RIP}) .
$$

In addition, every pseudo effect algebra with $(\mathrm{RDP})_{2}$ is an interval in a unital

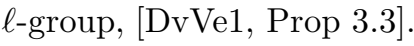

In an analogous way we can define the same Riesz Decomposition Properties for a po-group $G$, where instead of $E$ we deal with the positive cone $G^{+}$.

We recall that an $M V$-algebra is an algebra $\left(A ; \oplus,{ }^{*}, 0\right)$ of signature $\langle 2,1,0\rangle$, where $(A ; \oplus, 0)$ is a commutative monoid with neutral element 0 , and for all $x, y \in A$

(i) $\left(x^{*}\right)^{*}=x$,

(ii) $x \oplus 1=1$, where $1=0^{*}$,

(iii) $x \oplus\left(x \oplus y^{*}\right)^{*}=y \oplus\left(y \oplus x^{*}\right)^{*}$.

Sometimes it is used also a total binary operation $\odot$ defined by $a \odot b:=\left(a^{*} \oplus b^{*}\right)^{*}$.

If we define a partial addition, + , via $a+b$ is defined iff $a \leq b^{*}$, then $a+b=a \oplus b$, then $(A ;+, 0,1)$ is an effect algebra with $(\mathrm{RDP})_{2}, \mathrm{DvVe2}$, or equivalently a Phisymmetric effect algebra, $\mathrm{BeFo}$; and it is an interval in an Abelian unital $\ell$-group. Conversely, every lattice ordered effect algebra with (RDP) or equivalently, every effect algebra with $(\mathrm{RDP})_{2}$ is in fact an MV-algebra.

\section{Signed Measures and Jordan Signed Measures on Pseudo Effect Algebras}

In the present section, we describe the lattice structures of the set of Jordan signed measures on a pseudo effect algebra satisfying (RDP). We show that it is either trivial or a nontrivial Dedekind complete Riesz space.

Let $E$ be a pseudo effect algebra. A signed measure on $E$ is any mapping $m$ : $E \rightarrow \mathbb{R}$ such that $m(a+b)=m(a)+m(b)$ whenever $a+b$ is defined in $E$. Then $m(0)=0$ and $m\left(a^{-}\right)=m\left(a^{\sim}\right)$ for each $a \in E$. A measure is a positive signed measure $m$, i.e. $m(a) \geq 0$ for $a \in E$. Every measure is monotone on $E$. A state on $E$ is any measure $s$ such that $s(1)=1$. Let $\mathcal{M}(E), \mathcal{M}(E)^{+}$, and $\mathcal{S}(E)$ be the sets of all signed measures, measures, and states on $E$, respectively. It is clear that $\mathcal{M}(E) \neq \emptyset$ whilst $\mathcal{S}(E)$ can be empty. On $\mathcal{M}(E)$ we introduce a weak topology of signed measures defined as follows: a net of signed measures, $\left\{m_{\alpha}\right\}$, converges weakly to a signed measure $m$ iff $\lim _{\alpha} m_{\alpha}(a)=m(a)$ for every $a \in E$. Then $\mathcal{M}(E)$ is a non-void compact Hausdorff topological space. Similarly, $\mathcal{S}(E)$ is a compact Hausdorff space that can be sometimes void. Moreover, $\mathcal{S}(E)$ is a convex set, i.e. if $s_{1}, s_{2} \in \mathcal{S}(E)$ and $\lambda \in[0,1]$, then $s=\lambda s_{1}+(1-\lambda) s_{2} \in \mathcal{S}(E)$. A state $s$ is extremal if from the property $s=\lambda s_{1}+(1-\lambda) s_{2}$ for some $s_{1}, s_{2} \in \mathcal{S}(E)$ and $\lambda \in(0,1)$, we conclude $s=s_{1}=s_{2}$. Let $\partial_{e} \mathcal{S}(E)$ denote the set of all extremal states on $E$. 
By the Krein-Mil'man Theorem, Goo, Thm 5.17], every state on $E$ is a weak limit of a net of convex combinations of extremal states. Hence, $\mathcal{S}(E) \neq \emptyset$ iff $\partial_{e} \mathcal{S}(E) \neq \emptyset$.

In what follows, we are inspired by the research in [Goo, pp. 37-41], where it was done for Abelian po-groups.

A mapping $d: E \rightarrow \mathbb{R}$ is said to be subadditive provided $d(0)=0$ and $d(x+y) \leq$ $d(x)+d(y)$ whenever $x+y \in E$.

Proposition 3.1. Let $E$ be a pseudo effect algebra with (RDP) and let $d: E \rightarrow \mathbb{R}$ be a subadditive mapping. For all $x \in E$, assume that the set

$$
D(x):=\left\{d\left(x_{1}\right)+\cdots+d\left(x_{n}\right): x=x_{1}+\cdots+x_{n}, x_{1}, \ldots, x_{n} \in E, n \geq 1\right\}
$$

is bounded above in $\mathbb{R}$. Then there is a signed measure $m: E \rightarrow \mathbb{R}$ such that $m(x)=\bigvee D(x)$ for all $x \in E$.

Proof. The map $m(x):=\bigvee D(x)$ is a well-defined mapping for all $x \in E$. It is clear that $m(0)=0$ and now we are going to show that $m$ is additive on $E$.

Let $x+y \in E$ be given. For all decompositions

$$
x=x_{1}+\cdots+x_{n} \text { and } y=y_{1}+\cdots+y_{k}
$$

with all $x_{i}, y_{j} \in E$, we have $x+y=x_{1}+\cdots+x_{n}+y_{1}+\cdots+y_{k}$, that yields

$$
\sum_{i} d\left(x_{i}\right)+\sum_{j} d\left(y_{j}\right) \leq m(x+y) .
$$

Therefore, $u+v \leq m(x+y)$ for all $u \in D(x)$ and $b \in D(y)$. Since $\mathbb{R}$ is Dedekind complete, $\bigvee$ is distributive with respect to + :

$$
\begin{aligned}
m(x)+m(y) & =(\bigvee D(x))+m(y)=\bigvee_{u \in D(x)}(u+m(y)) \\
& =\bigvee_{u \in D(x)}(u+(\bigvee D(y)))=\bigvee_{u \in D(x)} \bigvee_{v \in D(y)}(u+v) \\
& \leq m(x+y) .
\end{aligned}
$$

Conversely, let $x+y=z_{1}+\cdots+z_{n}$, where each $z_{i} \in E$. Then (RDP) implies that there are elements $x_{1}, \ldots, x_{n}, y_{1}, \ldots, y_{n} \in E$ such that $x=x_{1}+\cdots+x_{n}$, $y=y_{1}+\cdots+y_{n}$ and $z_{i}=x_{i}+y_{i}$ for $i=1, \ldots, n$. This yields

$$
\sum_{i} d\left(z_{i}\right) \leq \sum_{i}\left(d\left(x_{i}\right)+d\left(y_{i}\right)\right)=\left(\sum_{i} d\left(x_{i}\right)\right)+\left(\sum_{i} d\left(y_{i}\right)\right) \leq m(x)+m(y),
$$

and therefore, $m(x+y) \leq m(x)+m(y)$ and finally, $m(x+y)=m(x)+m(y)$ for all $x, y \in E$ such that $x+y$ is defined in $E$, so that $m$ is a signed measure on $E$.

Let $X$ be a poset. A mapping $m: X \rightarrow \mathbb{R}$ is said to be (i) relatively bounded provided that given any subset $W$ of $X$ which is bounded (above and below) in $X$, the set $m(W)$ is bounded in $\mathbb{R}$, (ii) bounded if $m(X)$ is bounded in $\mathbb{R}$.

We recall that if $m$ is a signed measure on $E$, then $m$ is relatively bounded iff $m$ is bounded.

If $G$ is a po-group, any group homomorphism $m: G \rightarrow \mathbb{R}$ is said to be a signed measure on $G$. Of course, if $m \neq 0$ is a measure that is relatively bounded on $G \neq\{0\}$, then it is not bounded on $G$. 
Lemma 3.2. If $m$ is a signed measure on a unital po-group $(G, u)$, then $m$ is relatively bounded iff $m$ is bounded on the interval $[0, n u]$ for each $n \geq 1$. If, in addition, $(G, u)$ satisfies (RDP), then $m$ is relatively bounded iff $m$ is bounded on the interval $[0, u]$.

Proof. Indeed, one direction is clear, now suppose that $m$ is bounded on each interval [0,nu], and let $W$ be bounded in $G$. Then $W \subseteq[a, b]$ and for some $a, b \in G$. There is an integer $n \geq 1$ such that $-a+b \leq n u$. Then $[a, b]=a+[0,-a+b] \subseteq$ $a+[0, n u]$ and $m(W) \subseteq m(a)+m([0, n u]) \subseteq m(a)+[\alpha, \beta]=[m(a)+\alpha, m(a)+\beta]$ for some $\alpha, \beta \in \mathbb{R}$. This gives $m(W)$ is bounded in $\mathbb{R}$.

If, in addition, $(G, u)$ satisfies (RDP), then $[0, n u]=[0, u]+\cdots+[0, u]$. If $m([0, u])$ is bounded in $\mathbb{R}$, then $m([0, u]) \subseteq[\alpha, \beta]$ for some $\alpha, \beta \in \mathbb{R}$. Then $m([0, n u]) \subseteq$ $[n \alpha, n \beta]$.

Proposition 3.3. Let $E$ be a pseudo effect algebra with (RDP) and let $m: E \rightarrow \mathbb{R}$ be a signed measure. Then $m$ is relatively bounded if and only if $m=m_{1}-m_{2}$ for some measures $m_{1}, m_{2}$ on $E$.

Proof. Assume that $m=m_{1}-m_{2}$ for some two measures $m_{1}, m_{2} \in \mathcal{M}(E)^{+}$. If $W \subseteq[a, b]$ in $E$, then $m_{1}(W) \subseteq\left[m_{1}(a), m_{1}(b)\right]$ and $m_{2}(W) \subseteq\left[m_{2}(a), m_{2}(b)\right]$. Then $m_{1}(a)-m_{2}(b) \leq m_{1}(b)-m_{2}(a)$ and $m(W) \subseteq\left[m_{1}(a)-m_{2}(b), m_{1}(b)-m_{2}(a)\right]$ that proves that $m$ is relatively bounded.

Conversely, let $m$ be relatively bounded. If we set $d(x):=m(x) \vee 0$ for all $x \in E$, then $d(0)=0$. For all $x, y \in E$ such that $x+y$ is defined in $E$, we have

$$
d(x+y)=(f(x)+f(y)) \vee 0 \leq(f(x) \vee 0)+(f(y) \vee 0)=d(x)+d(y),
$$

so that $d$ is subadditive.

Let us define $D(x)$ by (3.1) for each $x \in E$. We assert that $D(x)$ is bounded above in $\mathbb{R}$. By the assumption, there are elements $a, b \in H$ such that $f([0, x]) \subseteq[a, b]$. Fix a decomposition $x=x_{1}+\cdots+x_{n}$ with $x_{i} \in E$ for each $i=1, \ldots, n$. By [Goo, Lem 1.21], we have

$$
\sum_{i=1}^{n} d\left(x_{i}\right)=\sum_{i=1}^{n}\left(m\left(x_{i}\right) \vee 0\right)=\left(\bigvee_{A \in 2^{n}}\left(\sum_{i \in A} m\left(x_{i}\right)\right)\right) \vee 0 .
$$

For all $A \in 2^{n}$, we have

$$
0 \leq \sum_{i \in A} x_{i} \leq x, \text { and } \sum_{i \in A} m\left(x_{i}\right)=m\left(\sum_{i \in A} x_{i}\right) \leq b .
$$

Hence, $d\left(x_{1}\right)+\cdots+d\left(x_{n}\right) \leq b \vee 0$, and consequently, $b \vee 0$ is an upper bound for $D(x)$ that proves the assertion.

By Proposition 3.1 there exists a signed measure $m_{1}$ on $E$ such that $m_{1}(x)=$ $\bigvee D(x)$ for all $x \in E$. Since $m_{1}(x) \geq d(x) \geq 0, m_{1}(x)$ is a measure, and $m_{1}(x) \geq$ $d(x) \geq m(x)$ for all $x \in E$. Hence, $m_{2}=m_{1}-m$ is a measure on $E$, too.

A signed measure $m$ on a pseudo effect algebra $E$ is said to be Jordan if $m$ can be expressed as a difference of two positive measures on $E$, and let $\mathcal{J}(E)$ be the set of all Jordan measures on $E$. It is clear that $\mathcal{J}(E)$ is nonempty because the zero mapping on $E$ belongs to $\mathcal{J}(E)$.

For example, if $1<\operatorname{dim} H<\infty$, then on $\mathcal{L}(H)$ there is a signed measure that is not Jordan, see e.g. [Dvu, 3.2.4], whilst if $\operatorname{dim}=\aleph_{0}$, then by the Dorofeev-Sherstnev Theorem, every $\sigma$-additive signed measure on $\mathcal{L}(H)$ is Jordan, Dvu, Thm 3.2.20]. 
Proposition 3.3 says that a signed measure $m$ on a pseudo effect algebra $E$ with (RDP) is Jordan iff $m$ is relatively bounded.

Given two signed measure $m_{1}, m_{2} \in \mathcal{M}(E)$, we define $m_{1} \leq^{+} m_{2}$ whenever $m_{2}-m_{1}$ is a positive measure. Then $\leq^{+}$is a partial order on $\mathcal{M}(E)$ and $\mathcal{M}(E)$ is an Abelian po-group with respect to this partial order.

Let $(G ;+, 0, \leq)$ be a po-group. A subgroup $H$ of $G$ is said to be convex if from $x \leq y \leq z$, where $x, z \in H$ and $y \in G$, we have $y \in H$. An $o$-ideal is any directed convex subgroup of $G$.

Proposition 3.4. Let $E$ be a pseudo effect algebra with (RDP), let $\mathcal{J}(E)$ be the set of all Jordan signed measures on $E$. Then $\mathcal{J}(E)$ is a nonempty o-ideal of the po-group $\mathcal{M}(E)$.

Proof. Due to Proposition $3.3, \mathcal{J}(E)$ equals the subgroup of $\mathcal{M}(E)$ generated by the positive measures. Therefore, $\mathcal{J}(E)$ is a directed subgroup of $\mathcal{M}(E)$.

Given $m_{1} \in \mathcal{M}(E)$ and $m_{2} \in \mathcal{J}(E)$ such that $0 \leq+m_{1} \leq^{+} m_{2}$, write $m_{2}=$ $m_{1}^{\prime}-m_{2}^{\prime}$ for some measures $m_{1}^{\prime}, m_{2}^{\prime} \in \mathcal{M}(E)^{+}$. Since $m_{1} \leq^{+} m_{2} \leq^{+} m_{1}^{\prime}$, we have $m_{1}=m_{1}^{\prime}-\left(m_{1}^{\prime}-m_{1}\right)$ with $m_{1}^{\prime}$ and $m_{1}^{\prime}-m_{1}$ positive measures, and hence, $m_{1} \in \mathcal{J}(E)$. This proves that $\mathcal{J}(E)$ is an o-ideal of $\mathcal{M}(E)$.

Theorem 3.5. Let $E$ be a pseudo effect algebra with (RDP).

(a) The group $\mathcal{J}(E)$ of all Jordan signed measures on $E$ is an Abelian Dedekind complete $\ell$-group.

(b) If $\left\{m_{i}\right\}_{i \in I}$ is a nonempty system of $\mathcal{J}(E)$ that is bounded above, and if $d(x)=\bigvee_{i} m_{i}(x)$ for all $x \in E$, then

$$
\left(\bigvee_{i} m_{i}\right)(x)=\bigvee\left\{d\left(x_{1}\right)+\cdots+d\left(x_{n}\right): x=x_{1}+\cdots+x_{n}, x_{1}, \ldots, x_{n} \in E\right\}
$$

for all $x \in E$.

(c) If $\left\{m_{i}\right\}_{i \in I}$ is a nonempty system of $\mathcal{J}(E)$ that is bounded below, and if $e(x)=\bigwedge_{i} f_{i}(x)$ for all $x \in E$, then

$$
\left(\bigwedge_{i} m_{i}\right)(x)=\bigwedge\left\{e\left(x_{1}\right)+\cdots+e\left(x_{n}\right): x=x_{1}+\cdots+x_{n}, x_{1}, \ldots, x_{n} \in E\right\}
$$

for all $x \in E$.

Proof. Let $t \in \mathcal{J}(E)$ be an upper bound for $\left\{m_{i}\right\}$. For any $x \in E$, we have $m_{i}(x) \leq$ $t(x)$, so that the mapping $d(x)=\bigvee_{i} m_{i}(x)$ defined on $E$ is a a subadditive mapping. For any $x \in E$ and any decomposition $x=x_{1}+\cdots+x_{n}$ with all $x_{i} \in E$, we conclude $d\left(x_{1}\right)+\cdots+d\left(x_{n}\right) \leq t\left(x_{1}\right)+\cdots+t\left(x_{n}\right)=t(x)$. Hence, $t(x)$ is an upper set for $D(x)$ defined by $(3.1)$.

Proposition 3.1 entails there is a signed measure $m$ on $E$ such that $m(x)=$ $\bigvee D(x)$. For every $x \in E$ and every $m_{i}$ we have $m_{i}(x) \leq d(x) \leq m(x)$ that gives $m_{i} \leq^{+} m$. The mappings $m-m_{i}$ are positive measures belonging bo $\mathcal{M}(E)^{+}$that gives $m \in \mathcal{J}(E)$. If $h \in \mathcal{J}(E)$ such that $m_{i} \leq^{+} h$ for each $i \in I$, then $d(x) \leq h(x)$ for any $x \in E$. As above, we can show that $h(x)$ is also an upper bound for $D(x)$, whence $m(x) \leq h(x)$ for any $x \in E$ that gives $m \leq^{+} h$. In other words, we have proved that $m$ is the supremum of $\left\{m_{i}\right\}_{i \in I}$, and its form is given by (b).

Applying the order anti-automorphism $z \mapsto-z$ in $\mathbb{R}$, we see that infima exist in $\mathcal{J}(E)$ for any bounded below system $\left\{m_{i}\right\}_{i \in I}$, and their form is given by (c). 
By Proposition 3.3. $\mathcal{J}(E)$ is directed, combining (b) and (c), we see that $\mathcal{J}(E)$ is an Abelian Dedekind complete $\ell$-group.

For finite joins and meets of Jordan signed measures, Theorem 3.5 can be reformulated as follows.

Theorem 3.6. If $E$ is a pseudo effect algebra with (RDP), then the group $\mathcal{J}(E)$ of all Jordan signed measures on $E$ is an Abelian Dedekind complete lattice ordered real vector space. Given $m_{1}, \ldots, m_{n} \in \mathcal{J}(E)$,

$$
\begin{aligned}
& \left(\bigvee_{i=1}^{n} m_{i}\right)(x)=\sup \left\{m_{1}\left(x_{1}\right)+\cdots+m_{n}\left(x_{n}\right): x=x_{1}+\cdots+x_{n}, x_{1}, \ldots, x_{n} \in E\right\} \\
& \left(\bigwedge_{i=1}^{n} m_{i}\right)(x)=\inf \left\{m_{1}\left(x_{1}\right)+\cdots+m_{n}\left(x_{n}\right): x=x_{1}+\cdots+x_{n}, x_{1}, \ldots, x_{n} \in E\right\}
\end{aligned}
$$

for all $x \in E$.

Proof. Due to Theorem 3.5. $\mathcal{J}(E)$ is an Abelian Dedekind complete $\ell$-group. It is evident that it is a Riesz space, i.e., a lattice ordered real vector space.

Take $m_{1}, \ldots, m_{n} \in \mathcal{J}(E)$ and let $m=m_{1} \vee \cdots \vee m_{n}$. For any $x \in E$ and $x=x_{1}+\cdots+x_{n}$ with $x_{1}, \ldots, x_{n} \in E$, we have $m_{1}\left(x_{1}\right)+\cdots+m_{n}\left(x_{n}\right) \leq m\left(x_{1}\right)+$ $\cdots+m\left(x_{n}\right)=m(x)$. Due to Theorem 3.5, given an arbitrary real number $\epsilon>0$, there is a decomposition $x=y_{1}+\cdots+y_{k}$ with $y_{1}, \ldots, y_{k} \in E$ such that

$$
\sum_{j=1}^{k} \max \left\{m_{1}\left(y_{j}\right), \ldots, m_{n}\left(y_{j}\right)\right\}>m(x)-\epsilon .
$$

If $k<n$, we can add the zero elements to the decomposition, if necessary, so that without loss of generality, we can assume that $k \geq n$.

We note that if $a, b \in E$ are given such that $a+b$ is defined in $E$, the elements $a^{\prime}, a^{\prime \prime} \in E$ such that $a+b=b+a^{\prime}$ and $b+a=a^{\prime \prime}+b$ are said to be (right and left) conjugates of $a$ by $b$. Since $\mathbb{R}$ is Abelian, for any $h \in \mathcal{J}(E), h\left(a^{\prime}\right)=h(a)=h\left(a^{\prime \prime}\right)$.

We decompose the set $\{1, \ldots, k\}$ into mutually disjoint sets $J(1), \ldots, J(n)$ such that

$$
J(i):=\left\{j \in\{1, \ldots, k\}: \max \left\{m_{1}\left(y_{j}\right), \ldots, m_{n}\left(y_{j}\right)\right\}=m_{i}\left(y_{j}\right)\right\} .
$$

Assume $J(1)=\left\{j_{t_{1}}, \ldots, j_{n_{1}}\right\}$. Then the element $x_{1}:=x_{j_{t_{1}}}+\cdots+x_{j_{n_{1}}}$ is defined in $E$.

The element $x$ can be expressed in the form $x=x_{j_{t_{1}}}+\cdots+x_{j_{n_{1}}}+x_{j}^{\prime}+\cdots+x_{k}^{\prime}$, where $x_{j}^{\prime}, \ldots, x_{k}^{\prime} \in E$ are conjugates of $x_{j}, \ldots, x_{k}$.

In a similar way, let $J(2)=\left\{j_{t_{2}}, \ldots, j_{n_{2}}\right\}$ and let $x_{2}=y_{j_{t_{2}}}+\cdots+y_{j_{n_{2}}}$. Again, we can express $x$ in the form $x=x_{1}+x_{2}+y_{s}^{\prime \prime}+\cdots+y_{k}^{\prime \prime}$, where $y_{t}^{\prime \prime}$ 's are appropriate conjugates of $y_{s}^{\prime}, \ldots, y_{k}^{\prime}$. Processing in this way for each $J(i)=\left\{j_{t_{i}}, \ldots, j_{n_{i}}\right\}$, we define the element $x_{i}=c_{t_{t_{t_{i}}}}+\cdots+c_{t_{j_{i}}}$, where $c_{t_{j_{s}}}$ is an appropriate conjugate of the element $y_{t_{j_{s}}}$. Then $x=x_{1}+\cdots+x_{n}$, and

$$
\sum_{i=1}^{n} m_{i}\left(x_{i}\right)=\sum_{i=1}^{n} \sum_{j \in J(i)} m_{i}\left(y_{j}\right)=\sum_{i=1}^{k} \max \left\{m_{1}\left(y_{j}\right), \ldots, m_{n}\left(y_{j}\right)\right\}>m(x)-\epsilon
$$


This implies $m(x)$ equals the given supremum.

The formula for $\left(m_{1} \wedge \cdots \wedge m_{n}\right)(x)$ can be obtained applying the order antiautomorphism $z \mapsto-z$ holding in $\mathbb{R}$.

\section{Jordan Signed Measures}

Using the results of the previous Section, we will show some interesting properties of signed measures, like a Jordan decomposition, variation, etc.

Let $E$ be a pseudo effect algebra with (RDP), and let $0: E \rightarrow\{0\}$ be the zero signed measure. Then $\mathcal{J}(E)$ is a nontrivial Abelian $\ell$-group, i.e., $\mathcal{J}(E) \supset\{0\}$ iff $E$ admits at least one state. Moreover, 0 is the zero element of the $\ell$-group $\mathcal{J}(E)$. We recall that if $E$ is an effect algebra with (RDP), then $\mathcal{S}(E)$ is always nonempty.

We say that a convex subset $F$ of a convex set $K$ is a face if $x=\lambda x_{1}+(1-\lambda) x_{2} \in$ $F, 0<\lambda<1$, entail $x_{1}, x_{2} \in F$. For example, if $x$ is an extreme point of $K$, then the singleton $\{x\}$ is a face, and for any $X \subseteq K$, there is the face generated by $X$. Due to [Goo, Prop 5.7], the face $F$ generated by $X$ is the set of those points $x \in K$ for which there exists a positive convex combination $\lambda x+(1-\lambda) y=z$ with $y \in K$ and $z$ belonging to the convex hull of $X$.

In particular, the face of $K$ generated by a point $z \in K$ consists precisely of those points $x \in K$ for which there exists a positive convex combination $\lambda x+\beta y=z$ with $y \in K$.

Lemma 4.1. Let $E$ be a pseudo effect algebra and let $X$ be a subset of $\mathcal{S}(E)$. Then a state $s \in \mathcal{S}(E)$ belongs to the face generated by $X$ if and only if $s \leq^{+} \alpha$ for some positive constant $\alpha$ and some state $t$ in the convex hull of $X$.

Proof. If a state $s$ belongs to the face generated by $X$, by the note just before this lemma, there exists a positive convex combination $\lambda s+(1-\lambda) s^{\prime}=t$, where $s^{\prime} \in \mathcal{S}(E)$ and $t$ belongs to the convex hull of $X$. Then $\lambda s \leq^{+} t$ so that $s \leq^{+} t / \lambda$.

Conversely, if $s \leq^{+} \alpha t$ for some $\alpha>0$ and some state $t$ in the convex hull of $X$. Then $\alpha t-s$ is a measure, so that $\alpha t-s=\beta s^{\prime}$ for some $\beta \geq 0$. Now $s+\beta s^{\prime}=\alpha t$ and $1+\beta=s(1)+\beta s^{\prime}(1)=\alpha t(1)=\alpha$ that yields $1 / \alpha+\beta / \alpha=1$. This gives $0 \leq \lambda:=1 / \alpha \leq 1$ and $\lambda s+(1-\lambda) s^{\prime}=t$. Since $t$ belongs to the face generated by $X$, so does $s$.

Now we show that if $s_{1}$ and $s_{2}$ are two states on $E$, then $s_{1} \wedge s_{2}$ and $s_{1} \vee s_{2}$ are not necessarily states.

Proposition 4.2. Let $E$ be a pseudo effect algebra with (RDP). Let $F_{1}$ and $F_{2}$ be the faces generated by states $s_{1}$ and $s_{2}$, respectively, on $E$. The following statements are equivalent:

(i) $F_{1} \cap F_{2}=\emptyset$.

(ii) $s_{1} \wedge s_{2}=0$.

(iii) $s_{1} \vee s_{2}=s_{1}+s_{2}$.

(iv) Given $x \in E$ and any $\epsilon>0$, there exists $x_{1}, x_{2} \in E$ such that $x=x_{1}+x_{2}$ and $s\left(x \backslash x_{i}\right)<\epsilon$.

In particular, if $s_{1}$ and $s_{2}$ are two distinct extremal state on $E$, then $s_{1} \wedge s_{2}=0$.

Proof. (i) $\Rightarrow$ (ii). Assume that $s_{1}$ and $s_{2}$ belongs to mutually disjoint faces of $\mathcal{S}(E)$. If $s_{1} \wedge s_{2}>0$, there is a state $s$ and a real number $\alpha>0$ such that $s_{1} \wedge s_{2}=\alpha s$. Then $\alpha s \leq^{+} s_{1}$ and $\alpha s \leq^{+} s_{2}$ and $s \leq^{+} s_{1} / \alpha$ and $s \leq^{+} s_{2} / \alpha$. Lemma 4.1 implies $s$ 
belongs to the face generated by $s_{1}$ and as well to the one generated by $s_{1}$ that is absurd, so that $s_{1} \wedge s_{2}=0$.

(ii) $\Rightarrow$ (i). Let $F_{1}$ and $F_{2}$ be the faces generated by $s_{1}$ and $s_{2}$, respectively. We state that $F_{1} \cap F_{2}=\emptyset$. If not, there is a state $s \in F_{1} \cap F_{2}$ and by Lemma 4.1 $s \leq^{+} \alpha_{1} s_{1}$ and $s \leq^{+} \alpha_{2} s_{2}$. If $\alpha=\max \left\{\alpha_{1}, \alpha_{2}\right\}$, then $s \leq^{+} \alpha s_{1}$ and $s \leq^{+} \alpha s_{2}$ and $s / \alpha \leq^{+} s_{1}, s_{2}$ and therefore, $s / \alpha \leq^{+} s_{1} \wedge s_{2}=0$ that gives a contradiction.

(i) $\Leftrightarrow$ (iii). It follows from the basic properties of $\ell$-groups, see e.g. [Fuc, C p.67], $\left(s_{1} \vee s_{2}\right)+\left(s_{1} \wedge s_{2}\right)=s_{1}+s_{2}$.

(ii) $\Rightarrow$ (iv). By Theorem 3.6 given $x$ and $\epsilon>0$, there are $x_{1}^{\prime}, x_{2}^{\prime} \in E$ such that $x=x_{2}^{\prime}+x_{1}^{\prime}$ and $s_{1}\left(x_{2}^{\prime}\right)+s_{2}\left(x_{1}^{\prime}\right)<\epsilon$. Then $x_{2}^{\prime}=x \backslash x_{1}^{\prime}$ and $x_{1}^{\prime}=x_{2}^{\prime} / x$, and $s_{1}\left(x_{2}^{\prime}\right)=s_{1}\left(x \backslash x_{1}^{\prime}\right)<\epsilon$ and $s_{2}\left(x_{1}^{\prime}\right)=s_{1}\left(x_{2}^{\prime} / x\right)=-s_{1}\left(x_{2}^{\prime}\right)+s_{1}(x)=s_{1}(x)-$ $s_{1}\left(x_{2}^{\prime}\right)=s_{1}\left(x \backslash x_{2}^{\prime}\right)<\epsilon$.

But $x=x_{1}^{\prime}+x_{2}^{\prime \prime}$, where $x_{2}^{\prime \prime}$ is a conjugate of $x_{2}^{\prime}$ by $x_{1}^{\prime}$. Then $s_{2}\left(x_{2}^{\prime \prime}\right)=s_{2}\left(x_{2}^{\prime}\right)$. If we set $x_{1}=x_{1}^{\prime}$ and $x_{2}=x_{2}^{\prime \prime}$, we have $s_{1}\left(x \backslash x_{1}\right)=s_{1}\left(x \backslash x_{1}^{\prime}\right)<\epsilon, s_{2}\left(x \backslash x_{2}\right)=$ $s_{2}(x)-s_{2}\left(x_{2}\right)=s_{2}(x)-s_{2}\left(x_{2}^{\prime \prime}\right)=s_{2}(x)-s_{2}\left(x_{2}^{\prime}\right)=s_{2}\left(x \backslash x_{2}^{\prime}\right)<\epsilon$ and $x=x_{1}+x_{2}$.

(iv) $\Rightarrow$ (ii). Given $\epsilon>0$ and $x \in E$, there is a decomposition $x=x_{1}+x_{2}$ such that $s_{i}\left(x \backslash x_{i}\right)<\epsilon / 2$ for $i=1,2$. Hence, $x=x_{2}+x_{1}^{\prime}$, where $x_{1}^{\prime}$ is a conjugate of $x_{1}$. If we set $y=x_{2}$ and $z=x_{1}^{\prime}$, then using $x_{1}=x \backslash x_{2}$ and $x_{2}=x_{1} / x$, we have $s_{1}(y)=s_{1}\left(x_{2}\right)=s_{1}\left(x_{1} / x\right)=s_{1}\left(x \backslash x_{1}\right)<\epsilon / 2$ and $s_{2}(z)=s_{2}\left(x_{1}^{\prime}\right)=s_{2}\left(x \backslash x_{2}\right)<$ $\epsilon / 2$, so that $s(y)+s(z)<\epsilon$. By Theorem 3.6, this means $s_{1} \wedge s_{2}=0$.

Finally, if $s_{1}$ and $s_{2}$ are two distinct extremal states, then the singletons $\left\{s_{1}\right\}$ and $\left\{s_{2}\right\}$ are mutually disjoint faces. Hence, $s_{1} \wedge s_{2}=0$.

Proposition 4.3. Let $s_{1}, s_{2}$ be two states on a pseudo effect algebra $E$ with (RDP). Then $s_{1} \wedge s_{2} \in \mathcal{S}(E)$ if and only if $s_{1}=s_{2}$ and if and only if $s_{1} \vee s_{2}$ is a state.

Given $\lambda \in[0,1]$, let $s_{\lambda}:=\lambda s_{1}+(1-\lambda) s_{2} \in \mathcal{S}(E)$. Then $s_{1} \wedge s_{2}=\bigwedge\left\{s_{\lambda}: \lambda \in\right.$ $[0,1]\} \in \mathcal{M}^{+}(E)$.

Proof. Let $s=s_{1} \wedge s_{2} \in \mathcal{S}(E)$. Then $s \leq^{+} s_{1}$ and $s \leq^{+} s_{2}$. Therefore, $s_{i}-s$ is a positive measure. Since $s_{i}(1)-s(1)=0$ for $i=1,2$, we see that $s_{1}=s=s_{2}$. The converse statement is evident. The second equivalency follows from the $\ell$-group equality $\left(s_{1} \wedge s_{2}\right)+\left(s_{1} \vee s_{2}\right)=s_{1}+s_{2}$.

Let $s=s_{1} \wedge s_{2} \in \mathcal{M}^{+}(E)$. Given $\lambda \in[0,1]$, we have $\lambda s \leq^{+} \lambda s_{1}$ and $(1-\lambda) s \leq^{+}$ $(1-\lambda) s_{2}$ so that $s=\lambda s+(1-\lambda) s \leq^{+} \lambda s_{1}+(1-\lambda) s_{2}$. Hence $s \leq^{+} s_{0}:=\bigwedge\left\{s_{\lambda}\right.$ : $\lambda \in[0,1]\}$. If we set $\lambda=1$ or $\lambda=0$, we see that $s_{1}, s_{2} \in\left\{s_{\lambda}: \lambda \in[0,1]\right\}$. Therefore, $s_{0} \leq^{+} s$.

A signed measure $m$ on a pseudo effect algebra $E$ is $\sigma$-additive if, $\left\{a_{n}\right\} \nearrow$ a, i.e. $a_{n} \leq a_{n+1}$ for each $n \geq 1$ and $\bigvee_{n} a_{n}=a$, then $m(a)=\lim _{n} m\left(a_{n}\right)$. A measure $m$ is $\sigma$-additive iff $a_{n} \searrow 0$ entails $m\left(a_{n}\right) \searrow 0$.

Proposition 4.4. If $m_{1}$ and $m_{2}$ are $\sigma$-additive measures on a pseudo effect algebra with (RDP), so are $m_{1} \vee m_{2}$ and $m_{1} \wedge m_{2}$.

Proof. Let $a_{n} \searrow 0$. Due to Theorem [3.6, $m_{1}\left(a_{n}\right)+m_{2}\left(a_{n}\right) \geq\left(m_{1} \vee m_{2}\right)\left(a_{n}\right) \geq 0$ so that $\left(m_{1} \vee m_{2}\right)\left(a_{n}\right) \searrow 0$. Similarly, $m_{1}\left(a_{n}\right) \geq\left(m_{1} \wedge m_{2}\right)\left(a_{n}\right) \geq 0$ and $\left(m_{1} \wedge\right.$ $\left.m_{2}\right)\left(a_{n}\right) \searrow 0$.

Theorem 3.6 allows us to define, for any Jordan signed measure $m$, its positive and negative parts, $m^{+}$and $m^{-}$, via

$$
m^{+}:=m \vee 0 \quad \text { and } \quad m^{-}:=-(m \wedge 0)
$$


Then $m=m^{+}-m^{-},(-m)^{+}=m^{-}$, and $(-m)^{-}=m^{+}$.

Theorem 3.6 says that

$$
m^{+}(a)=\sup \{m(x): x \leq a\} \quad \text { and } \quad m^{-}(a)=\inf \{m(x): x \leq a\}
$$

for each $a \in E$.

The decomposition $m=m^{+}-m^{-}$is said to be Jordan, and if $m=m_{1}-m_{2}$ for some positive measures $m_{1}, m_{2}$ on $E$, then $m^{+} \leq^{+} m_{1}$ and $m^{-} \leq^{+} m_{2}$. Moreover, we define an absolute value, $|m|$, of $m$ via

$$
|m|=m^{+}+m^{-} .
$$

Therefore, if $\mathcal{S}(E) \neq \emptyset$, every Jordan signed measure $m$ can be uniquely expressed in the form

$$
m=\alpha_{1} s_{1}-\alpha_{2} s_{2},
$$

where $\alpha_{1}, \alpha_{2}$ are real numbers and $s_{1}, s_{1}$ are states such that $\alpha_{1} s_{1}=m^{+}$and $\alpha_{2} s_{2}=m^{-}$, we call it a canonical Jordan decomposition of $m$.

The measures $m^{+}, m^{-}$and $|m|$ are sometimes called also an upper or positive variation, a lower or negative variation and a total variation of $m$, respectively.

Proposition 4.5. For any Jordan signed measure $m$ on a pseudo effect algebra $E$ with (RDP), we define a mapping $v_{m}: E \rightarrow \mathbb{R}$ by

$$
v_{m}(x):=\sup \left\{\left|m\left(x_{1}\right)\right|+\cdots+\left|m\left(x_{n}\right)\right|: x=x_{1}+\cdots+x_{n}, n \geq 1\right\} .
$$

Then $v_{m}=|m|$.

Proof. Let $x=x_{1}+\cdots+x_{n}$. Then $\left|m\left(x_{1}\right)\right|+\cdots+\left|m\left(x_{n}\right)\right| \leq|m|\left(x_{1}\right)+\cdots+$ $|m|\left(x_{n}\right)=|m|(x)$, so that $v_{m}(x) \leq|m|(x)$. Due to $(4.1), m^{+}(x), m^{-}(x) \leq v_{m}(x)$ for each $x \in E$. We assert that $v_{m}$ is subadditive, i.e., $v_{m}(x+y) \leq v_{m}(x)+v_{m}(y)$ whenever $x+y \in E$. Indeed, if $x+y=z_{1}+\cdots+z_{n}$, (RDP) entails that there are $x_{1}, \ldots, z_{n}, y_{1}, \ldots, y_{n} \in E$ such that $x=x_{1}+\cdots+z_{n}$ and $y=z_{1}+\cdots+z_{n}$. Then $\left|m\left(z_{1}\right)\right|+\cdots+\left|m\left(z_{n}\right)\right| \leq \sum_{i}\left|m\left(x_{i}\right)\right|+\sum_{i}\left|m\left(y_{i}\right)\right| \leq v_{m}(x)+v_{m}(y)$, so that $v_{m}(x+y) \leq v_{m}(x)+v_{m}(y)$.

According to (3.1), we define the set

$$
V_{m}(x)=\left\{v_{m}\left(x_{1}\right)+\cdots+v_{m}\left(x_{n}\right): x=x_{1}+\cdots+x_{n}\right\} .
$$

This set is bounded in $\mathbb{R}$, its upper bound is $|m(x)|$. Proposition 3.1 yields that the functional $V(x)=\sup V_{m}(x), x \in E$, is a positive measure on $E$. It is clear that $v_{m}(x) \leq V(x) \leq|m|(x)$ for each $x \in E$.

We show that $v_{m}=V$. Given $\epsilon>0$, there is a decomposition $x=x_{1}+\cdots+x_{n}$ such that $\sum_{i} v_{m}\left(x_{i}\right)>V(x)-\epsilon$. For any $i=1, \ldots, n$, there is a finite decomposition of each $x_{i}=\sum_{j} x_{i}^{j}$ such that $\sum_{j}\left|m\left(x_{i}^{j}\right)\right| \geq v_{m}\left(x_{i}\right)-\epsilon / n$. Therefore,

$$
\sum_{i=1}^{n} \sum_{j}\left|m\left(x_{i}^{j}\right)\right|>\sum_{i=1}^{n}\left(v_{m}\left(x_{i}\right)-\epsilon / n\right)=\sum_{i=1}^{n} v_{m}\left(x_{i}\right)-\epsilon>V(x)-2 \epsilon .
$$

This entails, $v_{m}(x) \geq V(x)-2 \epsilon$. Since $\epsilon$ was arbitrary, $v_{m}(x) \geq V(x)$, consequently, $v_{m}(x)=V(x)$ for any $x \in E$.

Since $m^{+} \wedge m^{-}=0$, the $\ell$-group properties imply $|m|=m^{+}+m^{-}=m^{+} \vee m^{-}$. Since $m^{+}, m^{+} \leq^{+} v_{m}=V \leq^{+}|m|$, we have $v_{m}=|m|$. 
Let $\left\{a_{n}\right\}$ be a sequence of elements of a pseudo effect algebra $E$ such that $b_{n}=$ $a_{1}+\cdots+a_{n}$ exists for each $n \geq 1$. If $a=\bigvee_{n} b_{n}$ is defined in $E$, we write $a:=$ $a_{1}+a_{2}+\cdots=\sum_{n} a_{n}$. Let $\left\{a_{n}\right\} \nearrow a$. If we set $a_{1}^{\prime}=a_{1}$ and $a_{n}^{\prime}=a_{n-1} / a_{n}$ for each $n \geq 2$, then $a_{1}^{\prime}+\cdots+a_{n}^{\prime}=a_{n}$ for each $n \geq 1$ and $a=\sum_{n} a_{n}^{\prime}$. Hence, a signed measure $m$ is $\sigma$-additive iff $a=\sum_{n} a_{n}$ entails $m(a)=\sum_{n} m\left(a_{n}\right)$.

A pseudo effect algebra is said to be monotone $\sigma$-complete if $a_{1} \leq a_{2} \leq a_{n} \leq \cdots$, then $a=\bigvee_{n} a_{n}$ is defined in $E$. We say that $E$ satisfies $\sigma$-(RDP) if $a_{1}+a_{2}=$ $b_{1}+b_{2}+\cdots$, then there are two sequences $\left\{c_{1 n}\right\}_{n}$ and $\left\{c_{2 n}\right\}$ such that $a_{i}=\sum_{n} c_{i n}$ for $i=1,2$ and $b_{n}=c_{1 n}+c_{2 n}$ for each $n \geq 1$.

Proposition 4.6. Let $E$ be a pseudo effect algebra with $\sigma$-(RDP). If $m$ is a $\sigma$ additive Jordan signed measure, so is $\mathrm{m}^{+}, \mathrm{m}^{-}$and $|\mathrm{m}|$.

Proof. Assume $\left\{a_{n}\right\} \nearrow a$. If we set $a_{1}^{\prime}=a_{1}$ and $a_{n}^{\prime}=a_{n-1} / a_{n}$ for each $n \geq 2$, then $a_{1}^{\prime}+\cdots+a_{n}^{\prime}=a_{n}$ for each $n \geq 1$ and $a=\sum_{n} a_{n}^{\prime}$. Then $m(a)=\sum_{n} m\left(a_{n}^{\prime}\right)$.

We show that $|m|=v_{m}$ is $\sigma$-additive. We have $v_{m}(a) \geq v_{m}\left(a_{n}\right)$ so that $v_{m}(a) \geq$ $\lim v_{m}\left(a_{n}\right)$. Now assume $a=x_{1}+\cdots+x_{k}$. The $\sigma$-(RDP) entails that there is $k$ many sequences $\left\{c_{j n}\right\}_{n}$ for $j=1, \ldots, k$ such that $x_{j}=\sum_{n} c_{j n}$ and $a_{n}^{\prime}=\sum_{j=1}^{k} c_{j n}$ for each $n \geq 1$. Check

$$
\begin{aligned}
\sum_{j=1}^{k}\left|m\left(x_{j}\right)\right| & =\sum_{j=1}^{k}\left|m\left(\sum_{n} c_{j n}\right)\right|=\sum_{j=1}^{k}\left|\sum_{n} m\left(c_{j n}\right)\right| \\
& \leq \sum_{n} \sum_{j=1}^{k}\left|m\left(c_{j n}\right)\right| \leq \sum_{n} v_{m}\left(a_{n}\right),
\end{aligned}
$$

so that $v_{m}(a) \leq \sum_{n} \leq \lim _{n} v_{m}\left(a_{n}\right)$ and $v_{m}=|m|$ is $\sigma$-additive. Because $m+2 m^{+}=$ $|m|$, we see that $m^{+}$is $\sigma$-additive, consequently, so is $m^{-}$.

Suppose that $E$ admits at least one state. Given a positive measure $m$ on $E$ with $m(1)>0$, let $\mathcal{J}(m)=[0, m]:=\left\{t \in \mathcal{J}(E): 0 \leq^{+} t \leq^{+} m\right\}$ be an interval in $\mathcal{J}(E)$. We can define on it an MV-structure by $s \oplus t:=(s+t) \wedge m, s \odot t:=\{s+t-m\} \vee 0$, and $s^{*}=m-s$ for all $s, t \in \mathcal{J}(m)$. Then $\left(\mathcal{J}(m) ; \oplus,{ }^{*}, 0\right)$ is an MV-algebra, where $m=0^{*}$ is the unit element of $\mathcal{J}(m)$.

The partial operation, + , on $\mathcal{J}(m)$, is defined as follows: $s+t$ is defined in $\mathcal{J}(m)$ iff $s+t \leq^{+} m$, or equivalently, it coincides with the restriction of the standard addition of the functions $s$ and $t$ belongs to $\mathcal{J}(m)$.

It is clear that the state space of $\mathcal{J}(m)$ is non-void. Let $a \in E$ be a fixed element. The mapping $\mu_{a}: \mathcal{J}(m) \rightarrow[0,1]$ defined by $\mu_{a}(s):=s(a), s \in \mathcal{J}(m)$, is a state on $\mathcal{J}(E)$.

Moreover, the system of states $\left\{\mu_{a}: a \in E\right\}$ is order-determining, i.e. $\mu_{a}(s) \leq$ $\mu_{a}(t)$ for all $a \in E$, implies $s \leq^{+} t$.

\section{Simplex Structure of Pseudo Effect Algebras and Integrals}

This is the main section of the paper. We show that if a pseudo effect algebra satisfies (RDP), then its state space is either empty or a non-empty simplex. This will allow represent states by standard integrals.

The following notions on convex sets can be found e.g. in [Goo. Let $K_{1}, K_{2}$ be two convex sets. A mapping $f: K_{1} \rightarrow K_{2}$ is said to be affine if it preserves all 
convex combinations, and if $f$ is also injective and surjective such that also $f^{-1}$ is affine, $f$ is said to be an affine isomorphism and $K_{1}$ and $K_{2}$ are affinely isomorphic.

We recall that a convex cone in a real linear space $V$ is any subset $C$ of $V$ such that (i) $0 \in C$, (ii) if $x_{1}, x_{2} \in C$, then $\alpha_{1} x_{1}+\alpha_{2} x_{2} \in C$ for any $\alpha_{1}, \alpha_{2} \in \mathbb{R}^{+}$. A strict cone is any convex cone $C$ such that $C \cap-C=\{0\}$, where $-C=\{-x: x \in C\}$. A base for a convex cone $C$ is any convex subset $K$ of $C$ such that every non-zero element $y \in C$ may be uniquely expressed in the form $y=\alpha x$ for some $\alpha \in \mathbb{R}^{+}$and some $x \in K$.

We recall that in view of [Goo, Prop 10.2], if $K$ is a non-void convex subset of $V$, and if we set

$$
C=\left\{\alpha x: \alpha \in \mathbb{R}^{+}, x \in K\right\},
$$

then $C$ is a convex cone in $V$, and $K$ is a base for $C$ iff there is a linear functional $f$ on $V$ such that $f(K)=1$ iff $K$ is contained in a hyperplane in $V$ which misses the origin.

Any strict cone $C$ of $V$ defines a partial order $\leq_{C}$ via $x \leq_{C} y$ iff $y-x \in C$. It is clear that $C=\left\{x \in V: 0 \leq_{C} x\right\}$. A lattice cone is any strict convex cone $C$ in $V$ such that $C$ is a lattice under $\leq_{C}$.

A simplex in a linear space $V$ is any convex subset $K$ of $V$ that is affinely isomorphic to a base for a lattice cone in some real linear space. A simplex $K$ in a locally convex Hausdorff space is said to be (i) Choquet if $K$ is compact, and (ii) Bauer if $K$ and $\partial_{e} K$ are compact, where $\partial_{e} K$ is the set of extreme points of $K$.

A simplex is a generalization of a classical simplex in $\mathbb{R}^{n}$, and we recall that no disc or no convex quadrilateral in the plane are not simplices.

Theorem 5.1. If $E$ is a pseudo effect algebra with (RDP), then either $\mathcal{S}(E)$ is empty or it is a nonempty Choquet simplex.

Proof. Assume that $\mathcal{S}(E)$ is nonempty. Then the positive cone $\mathcal{M}(E)^{+}=\mathcal{J}(E)^{+}$ of the Abelian Dedekind complete $\ell$-group $\mathcal{J}(E)$ consists of all positive measures on $E$, so that $\mathcal{J}(E)^{+}=\left\{\alpha s: \alpha \in \mathbb{R}^{+}, s \in \mathcal{S}(E)\right\}$. Since $\mathcal{S}(E)$ lies in the hyperplane $\{m \in \mathcal{J}(E): m(u)=1\}$ which misses the origin, $\mathcal{S}(E)$ is a base for $\mathcal{J}(E)^{+}$, and $\mathcal{S}(E)$ is a simplex. On the other hand, $\mathcal{S}(E)$ is compact, so that $\mathcal{S}(E)$ is a Choquet simplex.

We note that if $E$ is an effect algebra with (RDP) and $0 \neq 1$, then $E$ admits at least one state because then $E=\Gamma(G, u)$ for some unital Abelian interpolation po-group $(G, u)$; now it is enough to apply [Goo, Cor 4.4]. Hence, its state space is always a non-empty Choquet simplex. If an effect algebra $E$ does not satisfy (RDP), then its state space is not necessarily a simplex; for instance, this is the case for $E=\mathcal{E}(H), \operatorname{dim} H>2$. On the other hand, the state space of a commutative $\mathrm{C}^{*}$-algebra or the trace space of a general $\mathrm{C}^{*}$ are simplices, [AlSc, Thm 4.4, p. 7] or BrRo, Ex 4.2.6].

On the other hand, it is important to recall that according to a delicate result of Choquet Alf, Thm I.5.13], for any pseudo effect algebra $E, \partial_{e} \mathcal{S}(E)$ is always a Baire space in the relativized topology induced by the topology of $\mathcal{S}(E)$, i.e. the Baire Category Theorem holds for $\partial_{e} \mathcal{S}(E)$.

Remark 5.2. Theorem 5.1 was proved for a pseudo effect algebra that is an interval pseudo effect algebra, i.e., $E=\Gamma(G, u)$, for a unital po-group $(G, u)$ with (RDP). 
However, we do not know whether every pseudo effect algebra with (RDP) is an interval pseudo effect $\Gamma(G, u)$, where also $(G, u)$ satisfies (RDP), it was necessary to prove Theorem 5.1 in full details.

If a pseudo effect algebra $E$ satisfies (RDP $)_{2}$, then according to [Dvu3, Thm 4.4], the state space of $E$ is either the empty set or a nonempty Bauer simplex.

Example 5.3. There is a pseudo effect algebra $E$ with (RDP) but $(\mathrm{RDP})_{2}$ fails to hold in $E$ such that $\mathcal{S}(E)$ is a non-void Bauer simplex.

Proof. Let $\mathbb{Q}$ be the set of all rational numbers and let $G=\mathbb{Q} \times \mathbb{Q}$ be ordered by the strict ordering, i.e. $\left(g_{1}, g_{2}\right) \leq\left(h_{1}, h_{2}\right)$ iff $g_{1}<h_{1}$ and $g_{2}<h_{2}$ or $g_{1}=h_{1}$ and $g_{2}=h_{2}$. If we set $u=(1,1)$, then $E=\Gamma(G, u)$ is an effect algebra with (RDP) that is not a lattice. If $s_{0}(g, h):=h$ and $s_{1}(g, h):=g$, then $s_{0}$ and $s_{1}$ are unique extremal states on $E$, and every state $s$ is of the form $s=s_{\lambda}:=\lambda s_{1}+(1-\lambda) s_{0}$, $\lambda \in[0,1]$, for more details, see [BCD, Ex 4.2].

A pseudo effect algebra $E$ has the Bauer simplex property ((BSP) for short), if $\mathcal{S}(E)$ is a non-void Bauer simplex.

Let $K$ be a compact convex subset of a locally convex Hausdorff space. A mapping $f: K \rightarrow \mathbb{R}$ is said to be affine if, for all $x, y \in K$ and any $\lambda \in[0,1]$, we have $f(\lambda x+(1-\lambda) y)=\lambda f(x)+(1-\lambda) f(y)$. Let $\operatorname{Aff}(K)$ be the set of all continuous affine functions on $K$. Then $\operatorname{Aff}(K)$ is a unital po-group with the strong unit 1 which is a subgroup of the po-group $\mathrm{C}(K)$ of all continuous real-valued functions on $K$ (we recall that, for $f, g \in \mathrm{C}(K), f \leq g$ iff $f(x) \leq g(x)$ for any $x \in K$ ). In addition, $\mathrm{C}(K)$ is an $\ell$-group and the function 1 is its strong unit.

We note that if $E$ is a pseudo effect algebra such that $\mathcal{S}(E) \neq \emptyset$, given $a \in E$, let $\hat{a}: \mathcal{S}(E) \rightarrow[0,1]$ such that $\hat{a}(s):=s(a), s \in \mathcal{S}(E)$. Then $\hat{a} \in \operatorname{Aff}(\mathcal{S}(E))$.

If $K$ is a compact Hausdorff topological space, let $\mathcal{B}(K)$ be the Borel $\sigma$-algebra of $K$ generated by all open subsets of $K$. Let $\mathcal{M}_{1}^{+}(K)$ denote the set of all probability measures, that is, all positive regular $\sigma$-additive Borel measures $\mu$ on $\mathcal{B}(K)$. We recall that a Borel measure $\mu$ is called regular if each value $\mu(Y)$ can be approximated by closed subspaces of $Y$ as well by open subsets $O$ such that $Y \subseteq O$.

We recall that if $x \in K$, then the Dirac measure $\delta_{x}$ defined by $\delta(A):=\chi_{A}(x)$, $A \in \mathcal{B}(K)$, is a regular Borel probability measure.

For two measures $\mu$ and $\nu$ we define the Choquet equivalence $\sim$ defined by

$$
\mu \sim \lambda \quad \text { iff } \quad \int_{K} f \mathrm{~d} \mu=\int_{K} f \mathrm{~d} \lambda, f \in \operatorname{Aff}(K) .
$$

If $\mu$ and $\lambda$ are nonnegative regular Borel measures on a convex compact set $K$, we introduce for them the Choquet ordering defined by

$$
\mu \prec \lambda \quad \text { iff } \quad \int_{K} f \mathrm{~d} \mu \leq \int_{K} f \mathrm{~d} \lambda, f \in \operatorname{Con}(K),
$$

where $\operatorname{Con}(K)$ is the set of all continuous convex functions $f$ on $K$ (that is $f\left(\alpha x_{1}+\right.$ $\left.(1-\alpha) x_{2}\right) \leq \alpha f\left(x_{1}\right)+(1-\alpha) f\left(x_{2}\right)$ for $x_{1}, x_{2} \in K$ and $\left.\alpha \in[0,1]\right)$. Then $\prec$ is a partial order on the cone of nonnegative measures. The fact $\lambda \prec \mu$ and $\mu \prec \lambda$ implies $\lambda=\mu$ follows from the fact that $\operatorname{Con}(K)-\operatorname{Con}(K)$ is dense in $\mathrm{C}(K)$.

Moreover, for any probability measure $\lambda$ there is a maximal probability measure $\mu$ in Choquet's ordering such that $\mu \succ \lambda$, [Phe, Lem 4.1]. 
We recall that the Choquet ordering $\mu \prec \nu$ between two probability measures $\mu$ and $\nu$ roughly speaking means that $\nu$ is located further out than $\mu$ towards the set of extremal points where the convex function have large values, [AlSc, p. 8].

Theorem 5.4. Let $E$ be a pseudo effect algebra with (RDP) and with $\mathcal{S}(E) \neq \emptyset$. Let $s$ be a state on $E$. Let $\psi: E \rightarrow \operatorname{Aff}(\mathcal{S}(E))$ be defined by $\psi(a):=\hat{a}, a \in E$, where $\hat{a}$ is a mapping from $\mathcal{S}(E)$ into $[0,1]$ such that $\hat{a}(s):=s(a), s \in \mathcal{S}(E)$. Then there is a unique state $\tilde{s}$ on the unital po-group $(\operatorname{Aff}(\mathcal{S}(E)), 1)$ such that $\tilde{s}(\hat{a})=s(a)$ for any $a \in E$.

The mapping $s \mapsto \tilde{s}$ defines an affine homeomorphism from the state space $\mathcal{S}(E)$ onto $\mathcal{S}(\Gamma(\operatorname{Aff}(\mathcal{S}(E)), 1))$.

Proof. Since $E$ is a pseudo effect algebra such that $\mathcal{S}(E)$ is non-void, Theorem 5.1 asserts that $\mathcal{S}(E)$ is a nonempty Choquet simplex. We define $\widehat{E}:=\{\hat{a}: a \in E\} \subset$ $\operatorname{Aff}(\mathcal{S}(E))$ and let $\operatorname{Aff}(E)$ be the Abelian subgroup of $\operatorname{Aff}(\mathcal{S}(E))$ generated by $\widehat{E}$. Given $s \in \mathcal{S}(E)$, let $\tilde{s}$ be a mapping defined on the unital Abelian po-group with $(\operatorname{RDP})(\operatorname{Aff}(\mathcal{S}(E)), 1)$ such that $\tilde{s}(f):=f(s), f \in \operatorname{Aff}(\mathcal{S}(E))$. Then $\tilde{s}$ is a state on $(\operatorname{Aff}(\mathcal{S}(E)), 1)$.

By [Goo, Thm 7.1], the mapping $s \mapsto \tilde{s}$ is an affine homeomorphism between $\mathcal{S}(E)$ and $\mathcal{S}(\operatorname{Aff}(\mathcal{S}(E), 1)$.

Theorem 5.5. Let $E$ be a pseudo effect algebra with (RDP) having at least one state. Let $s$ be a state on $E$. Then there is a unique maximal regular Borel probability measure $\mu_{s} \sim \delta_{s}$ on $\mathcal{B}(\mathcal{S}(E))$ such that

$$
s(a)=\int_{\mathcal{S}(E)} \hat{a}(x) \mathrm{d} \mu_{s}(x), \quad a \in E .
$$

Proof. Due to Theorem 5.1 $\mathcal{S}(E)$ is a nonempty Choquet simplex. By Theorem 5.4, there is a unique state $\tilde{s}$ on $(\operatorname{Aff}(\mathcal{S}(E)), 1)$ such that $\tilde{s}(\hat{a})=s(a), a \in A$.

Applying the Choquet-Meyer Theorem, [Phe, Thm p. 66], we have

$$
f(s)=\int_{\mathcal{S}(E)} f(x) \mathrm{d} \mu_{s}, \quad f \in \operatorname{Aff}(\mathcal{S}(E)) .
$$

Since $\hat{a} \in \operatorname{Aff}(\mathcal{S}(E))$ for any $a \in E$, we have the representation given by (5.1).

Theorem 5.6. Let $E$ be a pseudo effect algebra with (BSP) and let $s$ be a state on $E$. Then there is a unique regular Borel probability measure, $\mu_{s}$, on $\mathcal{B}(\mathcal{S}(E))$ such that $\mu_{s}\left(\partial_{e} \mathcal{S}(E)\right)=1$ and

$$
s(a)=\int_{\partial_{e} \mathcal{S}(E)} \hat{a}(x) \mathrm{d} \mu_{s}(x), \quad a \in E .
$$

Proof. Due to Theorem 5.5, we have a unique regular Borel probability measure $\mu_{s} \sim \delta_{s}$ such that (5.1) holds. The characterization of Bauer simplices, Alf, Thm II.4.1], says that then $\mu_{s}$ is a unique regular Borel probability measure $\mu_{s}$ on $\mathcal{B}(\mathcal{S}(E))$ such that $(4.1)$ holds and $\mu_{s}\left(\partial_{e} \mathcal{S}(E)\right)=1$. Hence, (5.2) holds.

It is worthy to remark a note concerning formula (5.2) that if $\mu$ is any regular Borel probability measure, the right-hand side of formula (5.1) defines a state, say $s_{\mu}$, on $E$. But if $\mu\left(\partial_{e} \mathcal{S}(E)\right)<1$, then for $s_{\mu}$ there is another regular Borel probability measure $\mu_{0}$ such that $\mu_{0}\left(\partial_{e} \mathcal{S}(E)\right)=1$ and it represents $s_{\mu}$ via (5.2). 
For example, take $E$ from Example 5.3 The state space of $\mathcal{S}(E)$ is affinely homeomorphic with the real interval $[0,1]$. Let $\mu_{L}$ be the Lebesgue measure on $[0,1]$. Formula (5.1) for $\mu_{L}$ defines a state $s_{L}$ on $E$ such that if $a=(g, h) \in E$ and $s_{\lambda}(g, h)=\lambda g+(1-\lambda) h$, then $s_{L}(a)=\int_{0}^{1}(\lambda g+(1-\lambda) h) \mathrm{d} \mu_{L}(\lambda)=(g+h) / 2$. So that $s_{L}=\left(s_{0}+s_{1}\right) / 2$, but $\mu_{L}\left(\partial_{e} \mathcal{S}(E)\right)=0$. In other words, $s_{L}$ has two different representations by regular Borel probability measures via $(5.1)\left(\mu_{L}\right.$ and $\left(\delta_{0}+\delta_{1}\right) / 2$, only second one is described by Theorem 5.5) and uniquely via (5.2).

Corollary 5.7. Let $E$ be a pseudo effect algebra with (RDP) having at least one state. Let $m$ be a Jordan signed measure on $E$ and let $m=\alpha s_{1}-\beta s_{2}$ be its canonical Jordan decomposition. Then there are unique maximal regular Borel probability measures $\mu_{s_{1}} \sim \delta_{s_{1}}$ and $\mu_{s_{2}} \sim \delta_{s_{2}}$ on $\mathcal{B}(\mathcal{S}(E))$ such that for $\mu_{m}:=\alpha_{1} \mu_{s_{1}}-\alpha_{2} \mu_{s_{2}}$ we have

$$
m(a)=\alpha_{1} \int_{\mathcal{S}(E)} \hat{a}(x) \mathrm{d} \mu_{s_{1}}(x)-\alpha_{2} \int_{\mathcal{S}(E)} \hat{a}(x) \mathrm{d} \mu_{s_{2}}(x)=\int_{\mathcal{S}(E)} \hat{a}(x) \mathrm{d} \mu_{m}(x)
$$

for each $a \in E$.

Proof. Since $m(a)=\alpha_{1} s_{1}(a)-\alpha_{2} s_{2}(a)$, the statement follows from (4.2) and Theorem 5.5

Theorem 5.8. Let $E$ be a pseudo effect algebra with (BSP) such that $E$ has at least one state. Let $m$ be a Jordan signed measure on $E$ and and let $m=\alpha_{1} s_{1}-\alpha_{2} s_{2}$ be its canonical Jordan decomposition.

Then there are unique regular Borel probability measures $\mu_{s_{1}}, \mu_{s_{2}}$ on $\mathcal{B}(\mathcal{S}(E))$ such that $\mu_{s_{i}}\left(\partial_{e} \mathcal{S}(E)\right)=1$ for $i=1,2$ and for $\mu_{m}:=\alpha_{1} \mu_{s_{1}}-\alpha_{2} \mu_{s_{2}}$ we have

$$
m(a)=\alpha_{1} \int_{\partial_{e} \mathcal{S}(E)} \hat{a}(x) \mathrm{d} \mu_{s_{1}}(x)-\alpha_{2} \int_{\partial_{e} \mathcal{S}(E)} \hat{a}(x) \mathrm{d} \mu_{s_{2}}(x)=\int_{\partial_{e} \mathcal{S}(E)} \hat{a}(x) \mathrm{d} \mu_{m}(x)
$$

for each $a \in E$.

Proof. It follows from (4.2) and Theorem 5.6 .

\section{Conclusion}

We have extended the study of representing states on effect algebras by integrals that was started in the paper Dvu2 for states on pseudo effect algebras, quantum structures where the partial addition is not more assumed to be commutative.

Our research is based on methods of simplices and their application to state spaces. We have showed that every pseudo effect algebra that satisfies the same kind of the Riesz Decomposition Property, (RDP), is always a Choquet simplex, Theorem 5.1] This Theorem extends the result known for effect algebras with (RDP), see [Dvu1, Thm 5.1], for pseudo effect algebras with a stronger version, $(\mathrm{RDP})_{1}$, that is always an interval in a unital po-group with $(\mathrm{RDP})_{1}$, and for interval pseudo effect algebras with (RDP), see [Dvu3, Thm 4.3]. We note that we do not know whether every pseudo effect algebra with (RDP) is an interval in a unital po-group.

Finally, this result was applied to represent states on pseudo effect algebras with (RDP) by integrals through regular Borel probability measures, Theorem 5.5 and Theorem 5.6. 
It is important to make a finale remark that formulas (5.1) and (5.2) show that they are a bridge between the approach by de Finetti who was a propagator of probabilities as finitely additive measures, and the approach by Kolmogorov for whom a probability measure was a $\sigma$-additive measure, [Kol]. The mentioned formulas say by a way that these two approaches are equivalent.

\section{REFERENCES}

[Alf] E.M. Alfsen, "Compact Convex Sets and Boundary Integrals", Springer-Verlag, Berlin, 1971.

[AlSc] E.M. Alfsen, F.W. Schultz, "State Spaces of Operator Algebras", Birkhäuser, BostonBasel-Berlin, 2001.

[BeFo] M.K. Bennett, D.J. Foulis, Phi-symmetric effect algebras, Found. Phys. 25 (1995), 16991722 .

[BiNe] G. Birkhoff, J. von Neumann, The logic of quantum mechanics, Ann. Math. 37 (1936), 823-834.

[BrRo] O. Bratteli, D.W. Robinson, "Operator Algebras and Quantum Statistical Mechanics," Springer-Verlag, New York, Heidelberg, Berlin, 1979.

[BCD] D. Buhagiar, E. Chetcuti, A. Dvurečenskij, Loomis-Sikorski theorem and Stone duality for effect algebras with internal state, http://arxiv.org/abs/1006.0503

[Dvu] A. Dvurečenskij, "Gleason's Theorem and Its Applications", Kluwer Academic Publisher, Dordrecht/Boston/London, 1993, 325+xv pp.

[Dvu1] A. Dvurečenskij, States on pseudo MV-algebras, Studia Logica 68 (2001), 301-327.

[Dvu2] A. Dvurečenskij, Every state on interval effect algebra is integral, J. Math. Phys. 51 (2010), 083508-12. DOI: 10.1063/1.3467463

[Dvu3] A. Dvurečenskij, States on pseudo effect algebras and integrals, http://arxiv.org/submit/90550

[DvPu] A. Dvurečenskij, S. Pulmannová, "New Trends in Quantum Structures", Kluwer Acad. Publ., Dordrecht, Ister Science, Bratislava, 2000.

[DvVe1] A. Dvurečenskij, T. Vetterlein, Pseudoeffect algebras. I. Basic properties, Inter. J. Theor. Phys. 40 (2001), 685-701.

[DvVe2] A. Dvurečenskij, T. Vetterlein, Pseudoeffect algebras. II. Group representation, Inter. J. Theor. Phys. 40 (2001), 703-726.

[FoBe] D.J. Foulis, M.K. Bennett, Effect algebras and unsharp quantum logics, Found. Phys. 24 (1994), 1325-1346.

[Fuc] L. Fuchs, Partially Ordered Algebraic Systems, Pergamon Press, Oxford, London, NY, Paris, 1963).

[Gla] A.M.W. Glass "Partially Ordered Groups", World Scientific, Singapore, 1999.

[Goo] K.R. Goodearl, "Partially Ordered Abelian Groups with Interpolation", Math. Surveys and Monographs No. 20, Amer. Math. Soc., Providence, Rhode Island, 1986.

[Kol] A.N. Kolmogorov, "Grundbegriffe der Wahrscheinlichkeitsrechnung", Julius Springer, Berlin, 1933.

[KoCh] F. Kôpka, F. Chovanec, D-posets, Math. Slovaca 44 (1994), 21-34.

[Kro] T. Kroupa, Every state on semisimple MV-algebra is integral. Fuzzy Sets and Systems 157 (2006), 2771-2782.

[Pan] G. Panti, Invariant measures in free MV-algebras, Comm. Algebra 36 (2008), 2849-2861.

[Phe] R.R. Phelps, "Lectures on Choquet's Theorem", Van Nostrand, Princeton, 1965.

[Rav] K. Ravindran, On a structure theory of effect algebras, PhD thesis, Kansas State Univ., Manhattan, Kansas, 1996. 